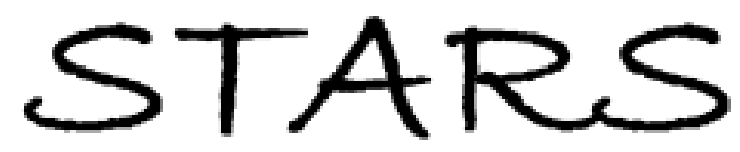

University of Central Florida

STARS

$1-1-1991$

\title{
130 Ps Recovery Of All-Optical Switching In A Gaas Multiquantum Well Directional Coupler
}

\author{
P. Li Kam Wa \\ University of Central Florida
}

A. Miller

University of Central Florida

J. S. Roberts

P. N. Robson

Find similar works at: https://stars.library.ucf.edu/facultybib1990

University of Central Florida Libraries http://library.ucf.edu

This Article is brought to you for free and open access by the Faculty Bibliography at STARS. It has been accepted for inclusion in Faculty Bibliography 1990s by an authorized administrator of STARS. For more information, please contact STARS@ucf.edu.

\section{Recommended Citation}

Wa, P. Li Kam; Miller, A.; Roberts, J. S.; and Robson, P. N., "130 Ps Recovery Of All-Optical Switching In A Gaas Multiquantum Well Directional Coupler" (1991). Faculty Bibliography 1990s. 269.

https://stars.library.ucf.edu/facultybib1990/269

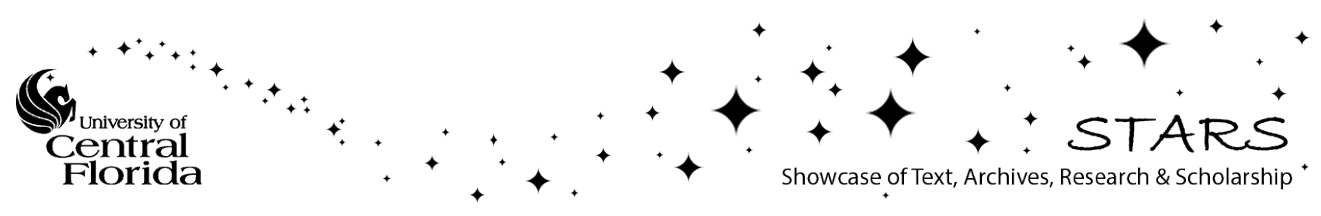




\section{0 ps recovery of all-optical switching in a GaAs multiquantum well directional coupler}

Cite as: Appl. Phys. Lett. 58, 2055 (1991); https://doi.org/10.1063/1.105008

Submitted: 02 January 1991 . Accepted: 04 March 1991 . Published Online: 04 June 1998

P. LiKamWa, A. Miller, J. S. Roberts, and P. N. Robson
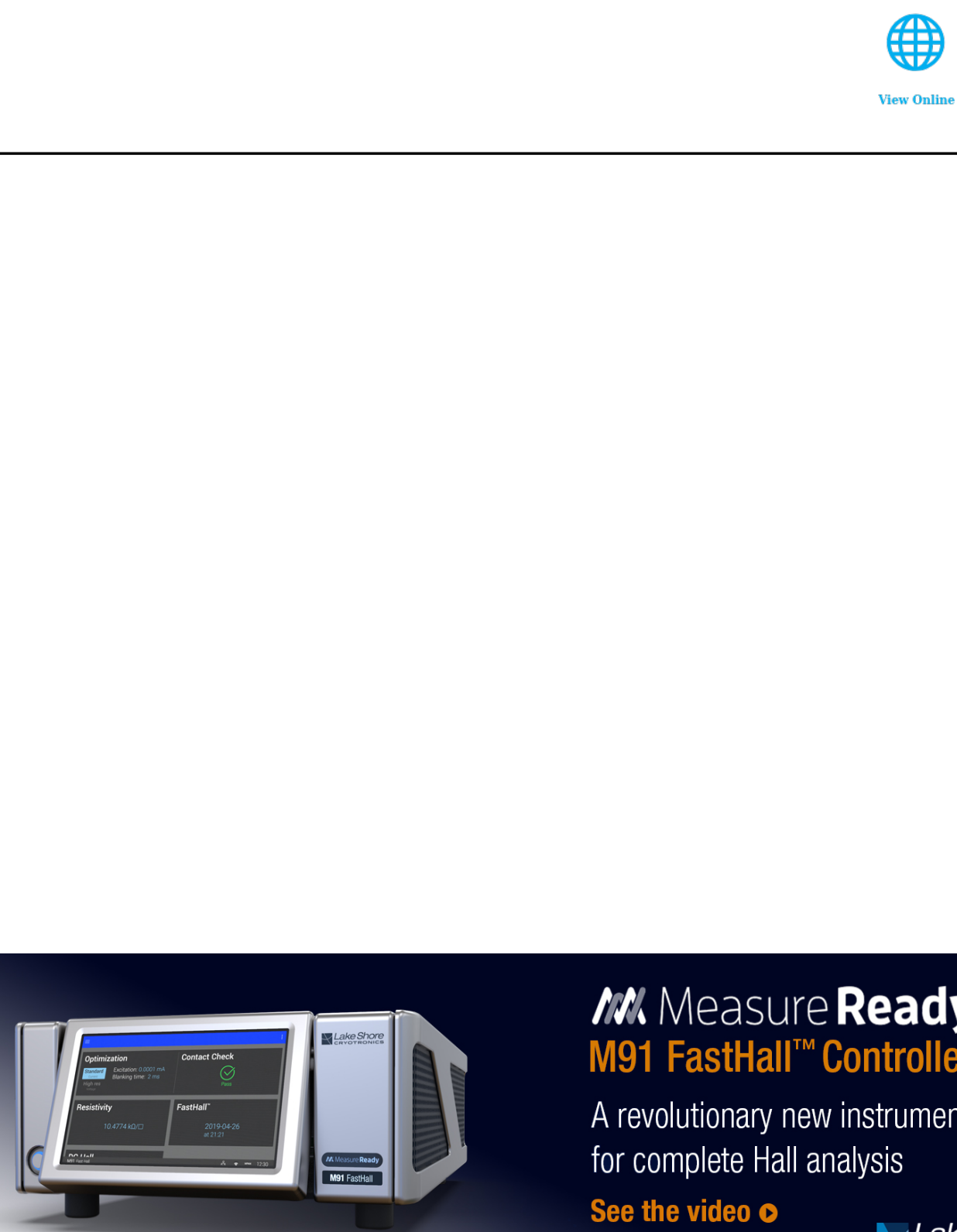

\section{Measure Ready M91 FastHall ${ }^{\text {TM }}$ Controller}

A revolutionary new instrument for complete Hall analysis

See the video 0

LakeShore
CRYOTRONICS 


\title{
130 ps recovery of all-optical switching in a GaAs multiquantum well directional coupler
}

\author{
P. LiKamWa and A. Miller \\ Center for Research in Electro-Optics and Lasers, University of Central Florida, Orlando, Florida 32826 \\ J. S. Roberts and P. N. Robson \\ Department of Electronic and Electrical Engineering, University of Sheffield, Sheffield S1 3JD, England
}

(Received 2 January 1991; accepted for publication 4 March 1991)

\begin{abstract}
A significantly improved recovery time of 130 ps has been achieved in an all-optical zero-gap directional coupler containing multiple quantum wells. The mechanism for the all-optical switching is due to free-carrier induced refractive nonlinearities at near-band-gap resonant frequencies. The large reduction in the switch recovery time was obtained by the application of an external dc bias which sweeps out the carriers from the quantum wells.

From our experimental results on the laser pulse width limited switch-up time of 2 ps, we deduce that the contribution of self-electro-optic effect nonlinearity is negligible in this case.
\end{abstract}

The nonlinear directional coupler first proposed by Jensen $^{1}$ is an integrated optical switch that can route signals all-optically into either of two output ports ${ }^{2}$ with potential applications in optical communication networks. The available nonlinear materials fall between two extremes. For example, the Kerr nonlinearity in fused quartz is very fast but because the nonlinearity is weak, a long interaction length is required. ${ }^{3}$ At the other extreme, many materials possess large thermal nonlinearities but the slow thermal response times limit the usefulness of these effects in waveguide devices. ${ }^{4}$ The electronically induced optical nonlinearities in semiconductor multiple quantum well (MQW) structures ${ }^{5}$ are also very large at frequencies close to resonance with the band-gap energy. Nonlinear refraction is induced by photogenerated free carriers that screen the excitons and shift the absorption edge by band filling. It is therefore evident that the response times of devices employing resonant optical nonlinearities in MQW structures are governed by the temporal and spatial dynamics of the photoinduced electrons and holes. The switch-up time is inherently governed by the temporal shape of the excite pulse, however the recovery time depends on the speed at which the carriers are removed. Normally the excess freecarrier population decays by the recombination of electrons with holes and is of the order of 5-10's ns in GaAs/ AlGaAs MQWs. In devices with a tightly focused geometry, such as waveguides, the carriers can also dissipate by spatially diffusing sideways due to the high concentration gradient. The time constant in this case is around $1 \mathrm{~ns}^{6}$ depending on the waveguide geometry.

In this work it is demonstrated that the application of an electric field perpendicular to the quantum wells rapidly sweeps the photocarriers out the wells and the waveguiding region such that the recovery of the device has been reduced to $\sim 100 \mathrm{ps}$. The use of carrier sweep-out has previously been used by Boyd et al. ${ }^{7}$ to improve on the switching time of symmetric self-electro-optic effect devices (SEEDs). In the present case, the switching time which is the rate of photogeneration of free carriers is only limited by the width of the laser pulses. On the other hand, the recovery of the directional coupler switch which depends on the removal of the carriers from the quantum wells is affected by the electric field in a similar manner as the switching time of a SEED.

The MQW structure studied in this work consisted of an undoped core guiding region that contains $25 \mathrm{GaAs}$ quantum wells each $100 \AA$ thick and separated by $100-\AA$ thick $\mathrm{Ga}_{0.75} \mathrm{Al}_{0.25} \mathrm{As}$ barrier layers. The top ( $p$ doped) and bottom ( $n$ doped) $\mathrm{Ga}_{0.75} \mathrm{Al}_{0.25} \mathrm{As}$ cladding regions are 1 and $3 \mu \mathrm{m}$ thick, respectively. The whole structure was grown by atmospheric pressure metalorganic vapor phase epitaxy on an $n$-type GaAs substrate. The room-temperature absorption spectrum revealed sharply resolved excitons with the heavy hole exciton absorption peak at 850 nm. $\mathrm{Zn} / \mathrm{Au} / \mathrm{Zn}$ and $\mathrm{Au}-\mathrm{Ge} / \mathrm{Ni}$ contacts were evap orated on the top cladding and the substrate, respectively, and subsequently alloyed in. Using conventional photolithography and reactive ion etching the top cladding was processed to leave $4-\mu \mathrm{m}$-wide ridges and the device was cleaved to a length of $500 \mu \mathrm{m}$. Through effective index loading, the MQW layer underneath the ridge confined light both in the vertical and lateral directions. The structure which is single moded in the vertical direction could support two lowest lateral modes to propagate simultaneously and beat with each other. The beating of these modes results in directional coupler-like action of the device.

The switch response characteristics were measured using a picosecond optical pump-probe technique. A cavitydumped Styryl-9 dye laser synchronously pumped by a frequency-doubled Nd:YAG laser provided picosecond pulses at a repetition rate of $7.6 \mathrm{MHz}$. These were split into pump and probe beams that could be temporally delayed with respect to each other. The pump beam passed through a half-wave plate while the probe beam was mechanically chopped. The two beams were then recombined on a beamsplitter and focused onto the same spot of the input facet of the device. The pump beam excited the TE modes of the waveguide while the probe beam excited the TM modes of the waveguide. A cross polarizer placed after the output collecting lens, ensured that only the TM-polarized chopped probe beam was monitored using a photodetector 


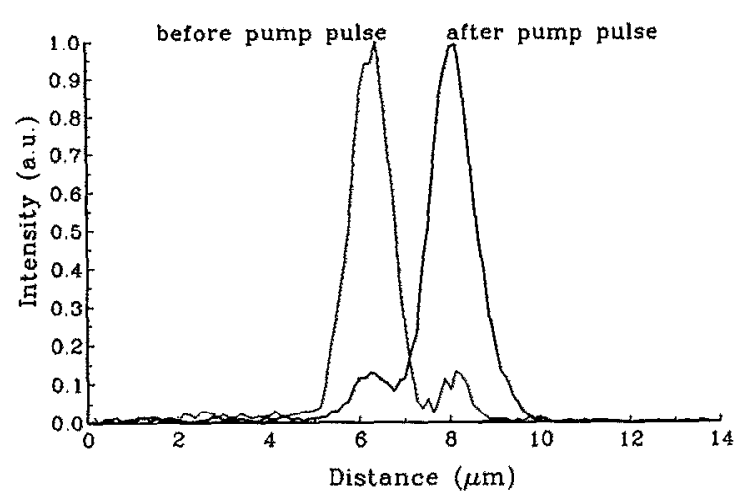

FIG. 1. TV line scans of the near-field probe output signal from the zero-gap waveguide coupler.

and lock-in amplifier. The near-field output pattern was observed using a TV camera and monitor. The wavelength of the laser was set to $860 \mathrm{~nm}$ where the absorption constant was estimated to be around $20 \mathrm{~cm}^{-1}$. A dc power supply and a $1 \mathrm{k} \Omega$ series resistor were connected across the top and bottom contacts with the negative terminal on the top ( $p$ contact).

By moving the probe delay stage such that the probe pulses arrived either before or after the corresponding pump pulses, directional switching of the probe due to the pump was observed. The switch on-off ratio which depends on the length of the device, could be optimized in two stages. First, the wavelength of the laser was adjusted (within the range where the nonlinearity was strong) for maximum contrast between the two output positions in the off-state (no-pump beam). The amplitude of the synchronized pump pulses was then adjusted for the best switchover response of the probe signal. TV line intensity scans of the output profiles with the probe pulses arriving just before and after the pump pulses are shown in Fig. 1.

The switch temporal response was obtained by measuring the output intensity from the "low" channel for each time increment as the probe timing was scanned past zero delay. Figure 2 shows the switch-up time to be around 2 ps which was limited by the pulse width of the laser. By performing a scan to long delays, the recovery time of the

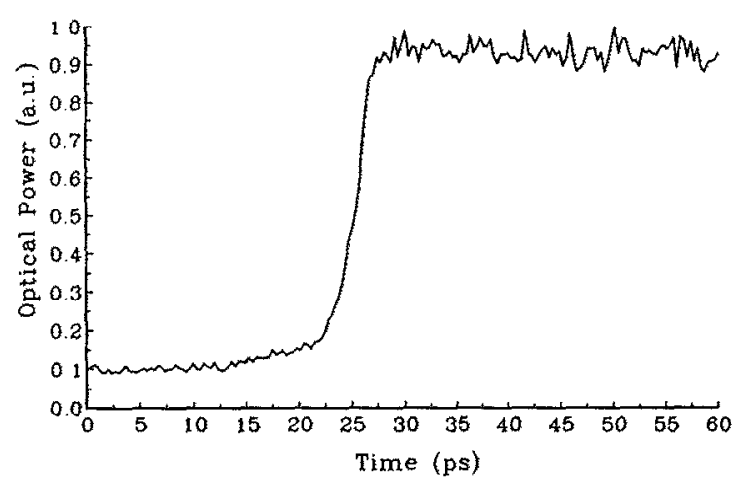

FIG. 2. Rise time of the probe signal being switched into the right (normally low) channel.

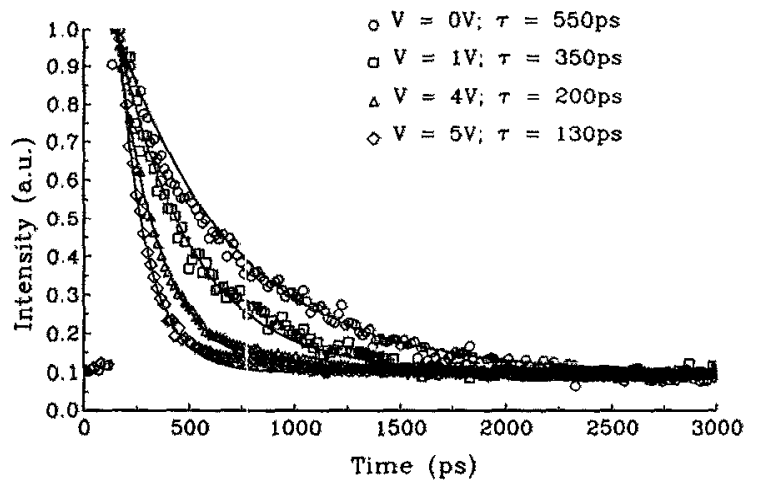

FIG. 3. Recovery of the switched probe signal as a function of applied voltage.

device was determined. A reverse bias was applied across the device and the measurement repeated for different bias voltages. As shown in Fig. 3, it was found that the time constant for the recovery consistently decreased from 550 ps under no external bias conditions to 130 ps when a reverse bias of $5 \mathrm{~V}$ (corresponding to $100 \mathrm{kV} / \mathrm{cm}$ ) was applied across the device. Previous measurements in a similar undoped MQW directional coupler gave a $1.5 \mathrm{~ns}$ recovery due to ambipolar diffusion of the carriers along the wells and out of the waveguiding regions. The faster recovery of 550 ps observed here in the unbiased condition may be due to the built-in field of the $p-i-n$ structure at zero applied volts.

From the observed fast switch-up time (laser pulse width limited), we can deduce that the refractive nonlinearity responsible for the switch is mainly due to freecarrier screening of band-gap absorption features instead of the self-induced electro-optic effect. The dynamics governing the recovery time is therefore only limited by the rate of carrier sweep out caused by the external field. The speed at which the carriers are swept out of the quantum wells to the cladding regions of the waveguide depends on both the field strength ${ }^{8}$ and the thickness of the barrier layers that separate the quantum wells from each other. In the case of thick barriers ( $100 \AA$ ) such as in the structure used for this work, the mechanism for the escape of the carriers at low fields is predominantly thermionic emission. At higher electric fields, ${ }^{9,10}$ the thermionic emission rate increases and tunneling occurs between the wells thus increasing the carrier sweep-out rate. By using thinner $\mathrm{Ga}_{0.75} \mathrm{Al}_{0.25} \mathrm{As}$ barriers $(\sim 35 \AA$; in the waveguide structure, the carriers could tunnel out of the wells much more quickly. It has been shown that under conditions of resonant tunneling, a photoconductive rise time of the order of $10 \mathrm{ps}$ can be achieved at low excitation levels. The sweep-out rate at higher excitation levels has yet to be determined. In these measurements, the minimum intensity required for the switch was unaffected by the applied electric field and is expected to remain the same as long as the recovery time stays longer than the pulse width of the laser.

In conclusion, it has been demonstrated that the application of an electric field across a $p-i \mathrm{MQW}-n$ structure can 
reduce the recovery time for a directional coupler optical switch by over an order of magnitude due to sweep out of the carriers that are generated by the optical pulse. This mechanism of carrier sweep out can also be applied to other all-optical devices that exploit resonant nonlinearities.

We acknowledge the support of the Florida Initiative in Advanced Microelectronics and Materials Program sponsored by the Defense Advanced Research Projects Agency.

'S. M. Jensen, IEEE J. Quantum Electron. QE-18, 1583 (1982).

${ }^{2}$ P. LiKamWa, J. E. Sitch, N. J. Mason, J. S. Roberts, and P. N. Robson, Electron. Lett. 21, 26 (1985).

${ }^{3}$ D. D. Gusovskii, E. M. Dianov, A. A. Maier, V. B. Neustruev, E. I.
Shklovsk $\overline{\mathrm{i}}$, and I. A. Shcherbakov, Sov. J. Quantum Electron. 15, 1523 (1985).

${ }^{4}$ J. S. Aitchison, J. D. Valera, A. C. Walker, S. Ritchie, P. M. Rodgers, P. McIlroy, and G. I. Stegeman, Appl. Phys. Lett. 51, 561 (1987).

${ }^{5}$ H. M. Gibbs, S. S. Tarng, J. L. Jewell, D. A. Weinberger, K. Tai, A. C. Gossard, S. L. McCall, and A. Passner, Appl. Phys. Lett. 41, 221 (1982).

${ }^{6}$ P. LiKamWa, A. Miller, C. B. Park, J. S. Roberts, and P. N. Robson, Appl. Phys. Lett. 57, 1846 (1990).

${ }^{7}$ G. D. Boyd, A. M. Fox, D. A. B. Miller, L. M. F. Chirovsky, L. A. D'Asaro, J. M. Kuo, R. F. Kopf, and A. L. Lentine, Appl. Phys. Lett. 57, 1843 (1990).

${ }^{8}$ R. J. Manning, P. J. Bradley, A. Miller, J. S. Roberts, P. Mistry, and M. A. Pate, Electron. Lett. 24, 854 (1988).

${ }^{9}$ G. Livescu, A. M. Fox, D. A. B. Miller, T. Sizer, W. H. Knox, A. C. Gossard, and J. H. English, Phys. Rev. Lett. 63, 438 (1989).

${ }^{10}$ A. M. Fox, D. A. B. Miller, G. Livescu, J. E. Cunningham, J. E. Henry, and W. Y. Yan, Appl. Phys. Lett. 57, 2316 (1990). 